\title{
Schulhausarchitekturen im physischen und sozialen Siedlungsraum
}

\author{
Jan Egger
}

Online publiziert: 3. August 2020

(C) Der/die Autor(en) 2020

Zusammenfassung Aus einer architektursoziologischen Perspektive werden Schulanlagen daraufhin analysiert, welche latenten, ,in Stein“ gehauenen Sinnstrukturen sie repräsentieren: Welche Bedeutungen kommt der Schule innerhalb der Siedlung zu? Welche sozialen Gruppen und wie werden diese über Architektur adressiert? Welche Normalitäten werden über Gebäude versinnbildlicht, und welche Strukturtransformationen lassen sich rekonstruieren? Es zeigt sich, dass in Schulbauten als „gebaute Staatlichkeit“ die Bauherrschaft dominiert. Schulhäuser sind für Kommunen auch ein Präsentationsmittel z. B. im Steuerwettbewerb. Damit beschreiben Schulhäuser auch den Sozialen Raum. Zudem zeigt sich eine soziale Ausweitung des Schulraums und damit einhergehend eine ,schulförmige“ Adressierung auch von Nicht-Schülern und Nicht-Schülerinnen.

Schlüsselwörter Schulhausarchitektur $\cdot$ Schulraum · Sozialraum · Architektursoziologie $\cdot$ Sozialisation über Artefakte $\cdot$ Schulförmigkeit

Dr. phil. J. Egger $(\bowtie)$

Pädagogische Hochschule, Institut Primarstufe, Professur Unterrichtsentwicklung und

Unterrerichtsforschung, Fachhochschule Nordwestschweiz FHNW, Hofackerstr. 30, 4132 Muttenz,

Schweiz

E-Mail: Jan.egger@fhnw.ch 


\title{
School building architecture in physical and social settlement areas
}

\begin{abstract}
The study analyses school buildings from an architectural sociological perspective to show which different latent meanings are "set in stone": What is the significance and sense of the school within the settlement? How are different social groups addressed via the architecture, and which groups are addressed? Which normalities are symbolized by school buildings and which structural transformations can be reconstructed? One finding shows that the building of schools as "built statehood" is dominated by the municipalities. School buildings are becoming an important form of representation in the tax competition between municipalities. So, school buildings are also defining the social space. In addition, there has been a social expansion of the school space and, as a result, a "school-like" addressing of non-students as well.
\end{abstract}

Keywords Architecture from school buildings - Social space $\cdot$ Architectural sociology $\cdot$ Socialization about artifacts $\cdot$ School-form

\section{Einleitung}

Jeder typische Raum wird durch typische gesellschaftliche Verhältnisse zustande gebracht, die sich ohne die störende Dazwischenkunft des Bewusstseins in ihm ausdrücken. (...) Die Raumbilder sind die Träume der Gesellschaft. Wo immer die Hieroglyphe irgendeines Raumbildes entziffert ist, dort bietet sich der Grund der sozialen Wirklichkeit. (Kracauer 1930)

Der Artikel untersucht aus einer architektursoziologischen Perspektive, wie sich Schulen in ihren Gebäuden gegenüber ihrer Umgebung positionieren und welche Bedeutungen damit einhergehen. Dabei soll insbesondere die Frage herausgearbeitet werden, wie der physisch-materiale Schulraum als „,gebaute Bildung“ mit dem sozialen Raum und dem Sozialisationsraum von Grundschülern verwoben ist. Das Konzept des Sozialen Raumes nach Bourdieu (1991) beschreibt eine hierarchisch strukturierte Gesellschaft, in der sich der soziale Raum auch in den physischen Raum einschreibt. Mit Bourdieu ist also danach zu fragen, welche symbolischen und manifesten Funktionen die Schule im Siedlungsraum einnimmt, die den sozialen Raum mitstrukturieren: Wie repräsentiert sich die Institution und Organisation Schule in ihrer Architektur gegenüber ,ihrem“ Siedlungsraum? Umgekehrt werden die Positionen der Subjekte - hier vor allem Grundschüler und -schülerinnen - im sozialen Raum auch durch die Bewegungen im physischen Raum einsozialisiert, die dessen Strukturen in einem stummen Mitlernen der Körper inkorporieren und internalisieren. Unter dieser Perspektive wird fokussiert, welche impliziten, sozialräumlich relevanten, formativen Sinn- und Bedeutungsstrukturen sich in Schulhäusern und -anlagen manifestieren: Wie werden Schüler und Schülerinnen, aber auch Nicht-Schüler und Nicht-Schülerinnen durch Architektur adressiert? Welche Strukturprinzipien prägen den Schulraum, der einen zentralen Sozialisationsraum von Grundschülern und -schülerinnen darstellt? 
Der Beitrag beruht auf einer empirischen Analyse von Schulanlagen und -architekturen (Egger 2019). Die architektursoziologische Perspektive geht davon aus, dass Architekturen einen konstitutiven Charakter haben (vgl. Fischer 2017). Gebäude sind ein affines Abbild sozialer Strukturen und wirken für die Individuen zugleich als Einflussstruktur handlungsleitend, indem sie vorstrukturierte Möglichkeitsräume eröffnen: bei Grundschulen beispielsweise als materialisierte Objektivationen der sekundären Sozialisation, die kontrastierend zum familialen Sozialisationsraum steht. Denn mit dem Schuleintritt wird die kindliche Sozialisation auch räumlich entzweit, was sich in unterschiedlich strukturierten Raumordnungen spiegelt und theoriesprachlich als primäre und sekundäre Sozialisation gefasst wird. Schulbauten stellen daher in ihren Architekturen akzentuiert nicht nur die funktionalen Vorstellungen vom Schülersein und von schulspezifischen Praktiken wie Unterricht besonders konturiert dar. Sie versinnbildlichen immer auch eine kulturund zeitgebundene „Normalität“. Dies gilt für Schulen generell. Bei Primarschulen der Schuleingangsstufe ${ }^{1}$ zeigen sich die Kontraste besonders deutlich, da die sozialisatorischen und erziehenden Effekte über Raum und Architekturen umso größer sind, je jünger Schülerinnen und Schüler sind. Zudem sind gerade die ersten Jahre der Grundschule noch durch die Internalisierung der Schuldisziplin (Durkheim 2003) als Mittel geprägt, die Kinder zum Lernen zu bringen. In Bezug auf die Gestaltung des sozialen Raumes einer Kommune sind die Grundschulen aussagekräftig, da sie für junge Familien mitunter ein Entscheidungskriterium der Wohnsitznahme darstellen.

Der Beitrag ist wie folgt gegliedert: Zuerst wird auf die architektursoziologischen Prämissen und das methodische Vorgehen eingegangen, die sich bei der Analyse von Architekturen ergeben. Danach werden in exemplarischer Hinsicht das Siedlungsbild, zwei ausgewählte Schulhäuser und der Schulhof einer Fallrekonstruktion ergebnisorientiert und illustrierend dargestellt. Der Fokus liegt auf den vorangestellten Fragen, wie sich der soziale und der physische Raum verweben, welche „soziale Wirklichkeit“, aber auch welche „gesellschaftlichen Träume“ sich aus den Raumbildern lesen lassen, wie dies Kracauer im Eingangszitat festhält.

\section{Architektursoziologische Prämissen und methodisches Vorgehen}

In einer strukturalen Architektursoziologie werden Gebäude als Sesshaftigkeit eines Kollektivs rekonstruiert (Schmidtke 2006). Als „Sitz“ der Schule stellen Gebäude die Innen-Außen-Abgrenzung des Kollektivs dar, womit auch Regeln z. B. der In- und Exklusion an Umfriedungen, an Schulplätze, -häuser und -zimmer gebunden sind. Als Außenfunktion repräsentieren Schulgebäude die Institution und Organisation Schule. Schulgebäude repräsentieren auch die Bauherrschaft und geben damit - was die Primarschule ${ }^{2}$ betrifft - der kommunalen Staatlichkeit ein „Gesicht“. Dadurch haben Schulgebäude auch kulturgeschichtlich einen enorm hohen Symbolwert, da

\footnotetext{
1 Die Primarstufe entspricht der deutschen Grundschule.

${ }^{2}$ Für die Gebäude der Primarstufe sind im subsidiären Politiksystem der Schweiz die Gemeinden zuständig.
} 
sie wie ,kaum ein anderer Gebäudetyp (...) den Wandel von politischen Systemen, sozialen und kulturellen Strukturen“ (Schäfers 2010, S. 37) illustrieren. Schulgebäude haben auch eine formative Wirkung in sozialräumlicher Hinsicht, indem sie für die Genese und Entwicklung von Siedlungsgebieten relevant sind, wie dies in der nachfolgenden Analyse gezeigt wird.

Neben der Repräsentationsfunktion gegen Außen haben Gebäude immer auch einen Auftrag. Als Binnenfunktion der Gebäude verkörpern Schulhäuser über das „Raumprogramm“ - die Summe aller Räume - und das „Programm der Räume“ eine explizite und implizite Theorie der Schule. Die Raum(an)ordnungen stellen eine Situationsdefinition her, in welche eine pädagogische Ordnung über örtlich gebundenen Rollenerwartungen und spezifische Handlungsweisen eingeschrieben ist.

Methodologisch werden Architekturen unter der Perspektive des Sitzes eines Kollektivs und als Repräsentation der Bewohnenden und der Bauherrschaft gesehen. Damit stellen sie für die einzelnen Akteure im Schulhaus eine Einflussstruktur dar, die jeweils einen vorkonfigurierten Handlungsraum eröffnet und vorstrukturiert, indem physisch-material und über die Schaffung einer Atmosphäre Situationsdefinitionen erzeugt werden, an welche Rollenerwartungen gebunden sind und die die Beziehungen der Akteure vorstrukturieren. Sozialisationstheoretisch ist dies relevant, da die mentalen Strukturen und damit auch die Stellung im sozialen Raum durch die Bewegung der Körper in ihrem (Sozial-)Raum habitualisiert und inkorporiert werden. Oder wie Alkemeyer (2011, S. 58) ausführt, erfolgt die Einverleibung des Sozialen ,in einem impliziten alltäglichen Mitlernen der Körper“ in den physischen und sozialen Räumen.

Die Untersuchung verfolgt methodisch ein quasi-archäologisches Vorgehen. Die Gebäude und architektonischen Artefakte stellen das alleinige Datenmaterial dar, was die genuine Originalität der Studie ausmacht. Es wird aus Raum(an)ordnungen, Gebäudeformationen und -platzierungen auf soziale Strukturen und Beziehungen geschlossen. Dazu wurden digitalisierte Luftbilder, Fotografien und Pläne von Schulanlagen sequenzanalytisch nach der Methode der Objektiven Hermeneutik ausgewertet (zur generellen Diskussion siehe Becker-Lenz et al. 2016; am Beispiel der Architektur Egger 2019; Schmidtke 2008). Die Objektive Hermeneutik hat zum Ziel, die latent wirkenden Sinn- und Bedeutungsstrukturen sichtbar zu machen. Die Architekturanalyse ist anspruchsvoll und aufwendig, da es sich um nicht-sprachlich und synchron wirkende Daten handelt. Aus Gründen des intersubjektiven Nachvollzuges müssen nicht-sprachliche Daten zunächst versprachlicht werden, was ,faktisch dargestellt, normativ beinhaltet und expressiv ausgedrückt wird“ (Barth 2004, S. 57). Danach zielt die Sequenzanalyse darauf ab, an jeder Sequenzstelle die objektiv gegebenen Möglichkeiten auszubuchstabieren, z. B. werden die möglichen Positionierungen eines Gebäudes oder einer Erschließung betrachtet und die damit einhergehenden Bedeutungen expliziert. Dadurch werden die regelgeleiteten Sinnstrukturen der faktisch realisierten Platzierung konturiert fassbar. Anzufügen ist, dass bei der Architekturanalyse zwangsläufig die longue durée fokussiert wird. Gebäude sind für eine lange Zeit gebaut. Daraus ziehen sie ihre stabilisierende soziale Funktion als „kollektives Gedächtnis“ (Halbwachs 1997), da ihre Bedeutungs- und Sinnstrukturen dauerhaft ,,in Stein gehauen“ werden. 
Die Ergebnisse werden abkürzend in ausgewählten Strukturprinzipien dargestellt: Zu Beginn wird die Positionalität der Schulgebäude im Siedlungsraum dargelegt, in welchen sich ein jeweils spezifisches Sendungsbewusstsein der Schule manifestiert. Im historischen Verlauf zeigt sich, dass sich der Schulraum in seinem Geltungsanspruch sukzessive entgrenzt und auch auf Nicht-Schüler ausdehnt. Danach werden die architektonischen Artefakte in ihren handlungsauffordernden Gesten fokussiert. Es reproduziert sich dabei ein Strukturprinzip, das unter dem Begriff ,Schulförmigkeit" gefasst wird. Schulförmigkeit meint, dass Architekturen und Dinge so arrangiert sind, dass sie genau monofunktional eine eindeutige Handlung evozieren und alle anderen verunmöglichen.

\section{Schulhausbau ist Siedlungsbau}

Exemplarisch wird die Argumentation an der Schulanlage der Schweizer Gemeinde Schmitten im Kanton Freiburg ausgeführt. Der Fall Schmitten wurde gewählt, da fünf Schulhäuser aus (bau)historisch unterschiedlichen Epochen vorhanden sind. Das älteste der Gebäude - heute Haus Grün genannt - wurde 1841 als Schulgebäude errichtet. Schulgebäude, die vor 1850 errichtet wurden, werden oft aus Untersuchungen ausgeschlossen, da sich der Gebäudetyp Schulhaus erst später entwickelt hat (z. B. Caruso 2003; Helfenberger 2016; Schneeberger 2005). Die Analyse des Hauses Grün scheint mir für die Beantwortung der Fragestellung jedoch besonders interessant. Die Schulanlage Schmitten wurde 2011 in einer Art und Weise neu gestaltet, wie dies den aktuellen, normativ geforderten Konzepten einer Öffnung des Schulraums entspricht. ${ }^{3}$ Schmitten erlaubt es daher, die Schule in ihren gesellschaftlich-demografischen, ökonomischen, sozial- und bildungspolitischen Entwicklungen zu rekonstruieren. Für den Artikel werden lediglich das älteste und das neuste Schulgebäude sowie der Außenraum betrachtet. (Abb. 1, 2 und 3).

Schmitten im Kanton Freiburg ist eine Siedlung im ländlich-agrarischen Gebiet. Alte, traditionelle Bauernhöfe sind als dezentral verstreute Gehöfte und Weiler auszumachen. Drei Gebiete prägen das Bild. Das Dorfzentrum in der Bildmitte (Abb. 4) liegt auf einer Anhöhe, auf welche zentripetal die Straßen zulaufen. Der alte Kern wurde etwa zeitgleich mit dem Bau der Eisenbahnlinie (Fertigstellung 1862) errichtet. Das Dorfzentrum besteht aus öffentlichen Gebäuden: Vom Bahnhof herkommend erkennt man eingangs des Dorfs die alten Schulhäuser, es folgt eine überproportional große Kirche (Baujahr 1898), das Gasthaus als Treffpunkt örtlicher Gemeinschaft und temporärer Aufenthalt für Fremde, das Gemeindehaus als gebaute Staatlichkeit sowie die Poststelle. Südöstlich des Zentrums ist ein größerer Perimeter, auf dem die neuen Schulhäuser verortet sind.

Nördlich davon - „,im Graben“ genannt - ergießt sich deltaförmig ein Quartier um den Bahnhof herum, das von Gewerbebauten und einfachen Wohnhäusern dominiert

\footnotetext{
3 Kernpunkt dieser normativen Setzung eines ,guten Schulraumes“ sind die Forderungen, dass Schulen auch Funktionen des ,community building“ übernehmen sollen, indem sie sich allen Schichten öffnen und einen inkludierenden Effekt, insbesondere für marginalisierte Bevölkerungsgruppen, einnehmen sollen (siehe z. B. Epstein und Salinas 2004).
} 


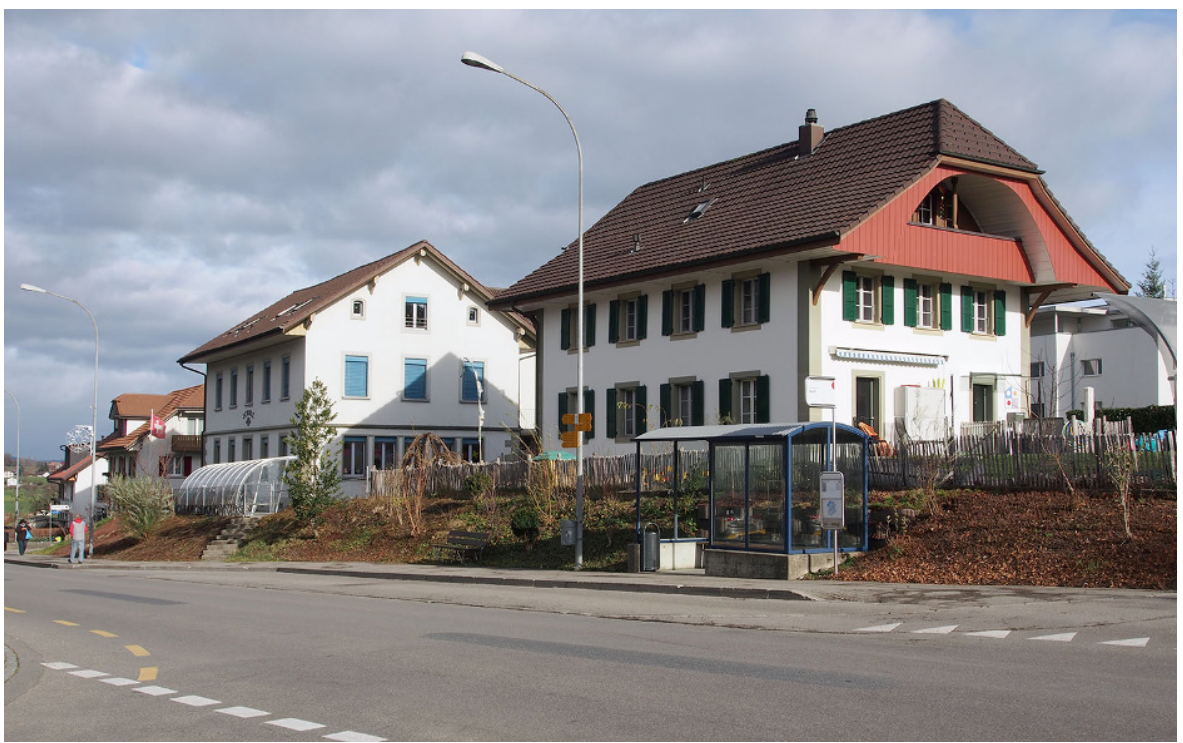

Abb. 1 Schulhaus Blau (hinten, 1891) (Schul-)Haus Grün (1841), Schmitten

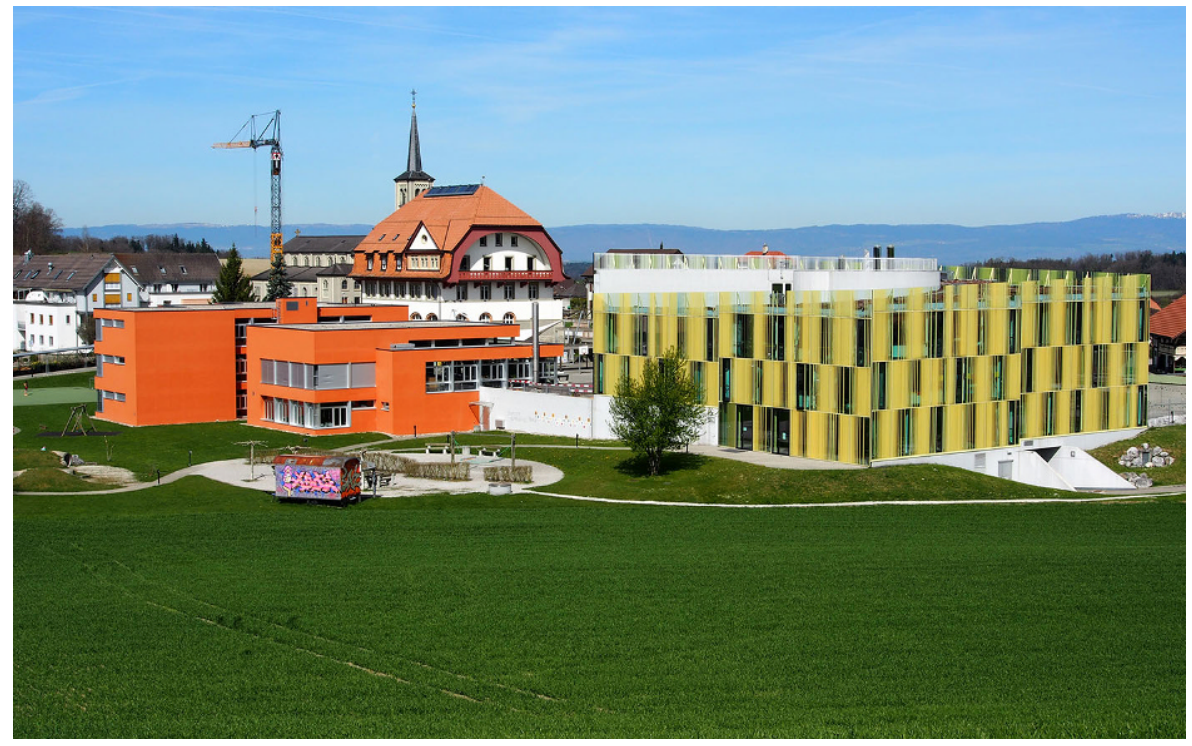

Abb. 2 Schulhäuser Orange (1970), Rot (hinten, 1931), Gelb (2011), Schmitten

wird. Südlich des Dorfzentrums liegt das neuste und mittlerweile größte Gebiet, „Barger“, welches in sich wiederum ein kleinräumiges, zentripetal ausgerichtetes Quartier bildet. Es sind vorwiegend von Grünflächen eingehegte (Doppel-)Einfamilienhäuser mit vielen Swimmingpools, was auf eine hohe Dichte an Familien und einen gehobenen Mittelstand verweist. 


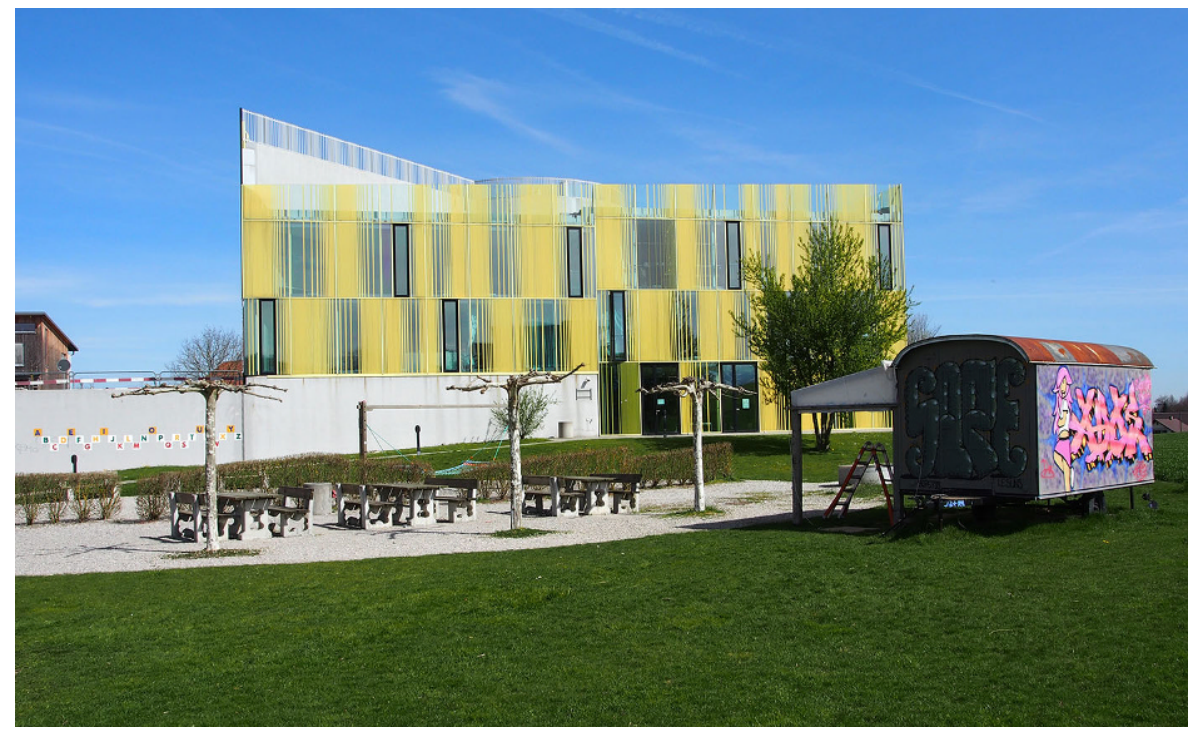

Abb. 3 Schulhaus Gelb, Schmitten

Abb. 4 Luftaufnahme Schmitten (Kanton Freiburg). (C) Google Earth 2015

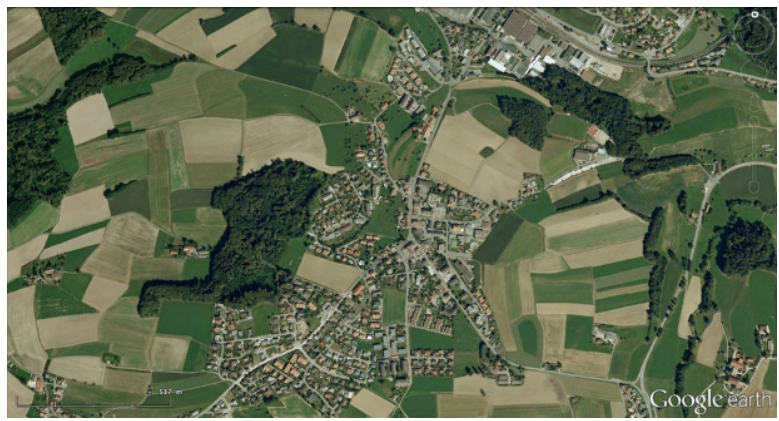

Im physischen Raum zeigt sich ein historisch emergiertes und transformiertes Spannungsverhältnis zwischen einer lose verstreuten, agrarisch orientierten autochthonen Bevölkerung, deren Zentrum eine Binnenorientierung darstellt. Dem steht eine außenorientierte, zugewanderte, (ehemals) industriell orientierte Bevölkerung im Bereich ,im Graben“ sowie in verschiedenen Siedlungen gegenüber, die in der zweiten Hälfte des 20. Jahrhunderts von neu zugezogenen Pendlern bewohnt werden. Die Lebensorte dieser sozialen Gruppen sind entzweit: eine binnenräumlich ausgerichtete Lebenswelt der Zuhause-Gebliebenen, zu denen insbesondere die Kinder im Quartier gehören, sowie eine ökonomische Außenorientierung eines oder beider Elternteile. Die divergierenden Strukturprinzipien stehen maßgeblich für die Umbrüche, welche die Siedlung Schmitten prägen und den demografischen, ökonomischen und sozio-kulturellen Wandel illustrieren und sich gleichsam in den Raum einschreiben. Sie stehen aber auch für ein Wachstumsprinzip der Siedlung Schmitten, im Zuge dessen im 19. Jahrhundert Industriearbeiter und im 20. Jahrhundert vor 
allem wohlhabende Mittelstandsfamilien zugezogen sind. Die These des Aufsatzes ist, dass die Schulhausarchitektur auch als Vehikel dieses Wachstums fungierte.

\section{1 (Schul-)Haus Grün: Schulraum als Konstitution eines differenten Sozial- und Sozialisationsraumes}

Bauhistorisch gesehen verdichtet der Bau der Schule den ehemals losen Weiler zu einem Dorf. Das Schulhaus und die später hinzukommende benachbarte Kirche transformieren die Siedlung zu einem öffentlichen Kumulationsraum sozialer Ausrichtung, wobei die Schule als Pionierbau rekonstruiert werden kann. ${ }^{4}$ Die soziale Stratifikation, die mit der ökonomischen, sozialen und politischen Transformation einhergeht, zeigt sich in der Verortung eines öffentlichen Raums im Zentrum, das aus funktional ausdifferenzierten Gebäuden besteht. Das örtliche Zentrum wird damit zu einem „stabilen Drehpunkt labiler Verhältnisse und Wechselwirkungen“, wie Simmel (1908, S. 473) die symbolischen Funktionen des Raums allgemein beschreibt. Das Schulhaus Grün nimmt dabei auch symbolisch in seiner Position eine besondere Stellung ein. Durch die Platzierung am Dorfeingang bildet das Schulgebäude einen weitherum sichtbaren Erkennungspunkt und bekommt eine identitätsstiftende Wirkung. Das Gebäude will gesehen werden und ist Ausdruck eines Sendungsbewusstseins. Denn das Gebäude ragt nicht nur manifest als singuläre Architektur aus der Siedlung heraus. Es kontrastiert in Morphologie, Baumaterialien und Raumaufteilung zu den umliegenden Bauernhäusern in revolutionär anmutender Weise. Die umliegenden Gehöfte sind für bäuerliche Großhaushalte erbaut, in welchen die Menschen in sippenhaften Haus- und Wirtschaftsgemeinschaften über Generationen lebten. In seiner Raumaufteilung und in seiner äußeren Gestalt ist das Schulhaus ein urbanes Stadthaus im Stil eines Handwerkerhauses, ohne dass ein Ökonomieteil ersichtlich ist. Das Haus hat die äußere und innere Struktur eines reinen Wohn- bzw. eines Miethauses. ${ }^{5}$ Damit verortet das Haus mitten in einer ländlich-ständischen Dorfstruktur eine urban geprägte, bürgerlich-kapitalistische Lebensweise.

Der Lehrberuf versinnbildlicht in seinem „Wohnhaus“ also eine Lebensart, die auf der Auftrennung von Wohnen und Arbeiten sowie von Familie und Betrieb beruht. Die bürgerliche Lebensweise setzt sich in den Räumlichkeiten und der Raumorganisation fort. Das Haus ist für einen Zweigenerationenhaushalt erbaut, und es hat eine Raumanordnung, die maßgeblich auf der Trennung von privaten und öffentlichen Räumen beruht. Damit werden einerseits die Kleinfamilie als Beziehungs- „Normalform“, aber andererseits auch die arbeitsteilig organisierten Geschlechterrollen dem Raum eingeschrieben. Für die Fragestellung von Interesse ist insbesondere die Auftrennung der Erziehungs- und Bildungsverhältnisse der Kinder durch die lokal verortete Schule. Dies geschieht in zeitlich-räumlicher, aber auch in sozialer Hinsicht, indem die kindliche Lebenswelt mit dem Bau des Schulhauses in diffe-

\footnotetext{
${ }^{4}$ Auch wenn Schulgebäude planerisch der demografischen Entwicklung fast immer hinterherhinken, wurden und werden sie oft auf der ,grünen Wiese“ erstellt. Sie sind damit Infrastruktur- und oft Pionierbauten in der Erschließung neuer Siedlungsgebiete.

${ }^{5}$ Quasi zeitgleich entstehen in Zürich die Escherhäuser, die als die ersten Mietshäuser in der Schweiz gelten.
} 
rent strukturierte (Sozialisations-)Räume fragmentiert wird: eine häuslich-familiale Erziehungsinstanz hier und eine schulisch-außerfamiliale dort. In den differenten Sozialisationsräumen gelten je unterschiedliche Regeln, Werte und Ordnungsprinzipien, sowohl was die expliziten Regeln betrifft als auch in Bezug auf die impliziten Regeln des tacit knowledge und des sogenannten hidden curriculum der Schule (Zinnecker 1975). Diese werden wesentlich perzeptiv erfahren und über die in Gebäuden angelegten Sinnstrukturen einverleibt.

Damit man sich in räumlichen Arrangements passend bewegen kann, müssen diese gelesen werden können (siehe Steets 2015, S. 185). Die Aneignung der Architektursprache und die an Architekturen gebundenen Normen und Werte werden implizit über die Bewegungen der Körper im Raum einverleibt und in Form von abgespeicherten Körpertechniken internalisiert und inkorporiert. So ist es kein Zufall, dass wir den Gebäudetyp Schule unmittelbar erkennen, unabhängig davon, aus welcher Zeit und in welchem Architekturstil ein Schulhaus erbaut ist. In dieser Perspektive scheint es mir bemerkenswert, dass das Haus Grün expressiv keinen Gebäudetyp Schule, sondern ein stattliches Wohnhaus darstellt. Auch wenn die Schule die Lebenswelt der Kinder in unterschiedliche Bildungs- und Sozialräume ,entzweit“, repräsentiert das Haus Grün als Wohnhaus der Lehrperson ideell noch - eine Einheit der Sozialisationsinstanzen, die ideengeschichtlich bei Pestalozzi als Schulwohnstube ausformuliert ist. Diese stellt eine geistige Werkstatt dar, eine „mit dem Geist und dem Wesen des häuslichen Lebens genau übereinstimmende Übungsanstalt“ (Pestalozzi zit. nach Perlick, 1969), die vor allem der Erziehung zur Sittlichkeit dient. Denn wie Pestalozzi (2010, S. 223) festhält: „Was der Mensch ist, das ist auch sein Haus. Und soweit als er selbst gebildet ist, nur soweit kann auch sein Haus bildend sein."

Die bürgerliche Lebensweise wird in der Nachbildung eines idealen Haushaltes quasi naturwüchsig einsozialisiert. Als „Lehrerwohnung“ repräsentiert das Schulhaus Grün auch die Lehrperson als Beamten und damit den jungen, liberalen 1841 noch kantonalen - Nationalstaat. Das Schulhaus Grün kann damit als Sinnbild und Werkzeug zugleich der Durchsetzung der neuen politischen, sozialen und ökonomischen Ordnung gesehen werden. Es gibt nicht nur der Schule, sondern auch der politischen Gemeinde im neu geschaffenen Dorfzentrum ein „Gesicht“. Dabei entfaltet es seine Wirkung nicht ,nur“ über die Wissensvermittlung im schulischen Unterricht, sondern genauso über die symbolische Sinnbildlichkeit und sozialisatorische Effektivität, indem über Raumordnungen auch die neue soziale und politische Ordnung einsozialisiert werden. Denn als Symbole betrachtet stehen Gebäude für Bedeutungsstrukturen, die ,die Wirklichkeit der Alltagswelt überschreiten“ und die wie ein ,abstraktes moralisches Prinzip, eine kollektive Idee oder gar die Anwesenheit von etwas ,Heiligem““ darstellen (Steets 2015, S. 192, Hervorhebung im Original).

Wenn das Schulhaus als Identitätsgenerator verstanden wird, kann festgehalten werden, dass Schulgebäude in ihren Gebäudeformationen und den Situierungen Pionierbauten einer gesellschaftlichen Strukturtransformation darstellen. Schulgebäude erzeugen eine sozialisatorische, soziale und auch biopolitische Effektivität, indem sie durch ihre Gehäuse Normalitätsvorstellungen der Sittlichkeit bzw. des ,richtigen“ Lebens, aber auch der Ökonomie und des Verhältnisses von Staat und Bürger 
dem Raum eingravieren. Dabei sind Grundschulgebäude sozialisatorisch besonders bedeutsam. Im krisenhaften Bildungsprozess, in dem es dem Kind gelingen muss, ,aus seinem Ich herauszutreten, um sich eine objektive Vorstellung der Wirklichkeit zu konstruieren“ (Piaget 2017, S. 57), sind sie der Aneignungsraum, der prototypisch den außerfamilialen Ort der Wirklichkeit bzw. der Vergesellschaftung darstellt.

So zeigt sich bereits am Haus Grün die Struktur einer formativen und repräsentativen Architektur, wenn dies auch sehr viel impliziter geschieht, als dies zwei Dekaden später bei den militärisch konnotierten, monumentalen Schulkasernen der Fall ist. Diese repräsentieren explizit gegen außen eine Herrschaftsarchitektur und stellen gegen innen einen formativen Lernraum her, der auch als „Überwachungs-, Hierarchisierungs- und Belohnungsmaschine" wirkt (Foucault 2012, S. 188, siehe auch Caruso 2003). Diese Schulkasernen verkörpert fast prototypisch auch das Schulhaus Blau. An dieser Stelle wird jedoch das neuste Schulhaus Gelb als größtmöglicher Kontrast fokussiert.

\subsection{Schulhaus Gelb: Exzentrische Architektur als Sinnbild und Gestaltung eines spezifischen sozialen Raumes}

Siedlungsexpansion und Bevölkerungsdruck haben eine sukzessive Erweiterung des Schulraums zur Folge. Im Prinzip stehen zwei Erweiterungsmodelle zur Auswahl: ein dezentrales Modell, das den Schülern und Schülerinnen in ihren kleinräumigen, bereits angeeigneten Sozialräumen ,entgegenkommt“, oder ein Modell mit zentral verorteten An- und Erweiterungsbauten. Für Grundschulkinder ist ein Modell radialer Zonen ihrer Bewegungsräume plausibel (siehe Kogler 2015) bzw. werden Schulhäuser in der Schweiz nach dem Prinzip eines „kurzen“ Schulweges meistens dezentralisiert erstellt. In Schmitten wurde ein zentrales Modell realisiert, wobei mit der Stratifikation des Schulsystems ein zweiter Schulstandort notwendig wurde. Wie in Abb. 5 erkennbar ist, wurden die Schulhäuser des 20. Jahrhunderts in einem eigenen Perimeter erstellt (Ausnahme ist der Erweiterungsbau des Schulhauses Blau am ,,alten“ Standort). Das 2011 erbaute Schulhaus Gelb liegt am Rand der Siedlung neben einem Acker. Es liegt abseits des Publikumsverkehrs der Hauptstraße.

Abb. 5 Alte (rote ausgezogene) und neue (grüne gestrichelte Linie) Schulhäuser, Schmitten. (C) Google

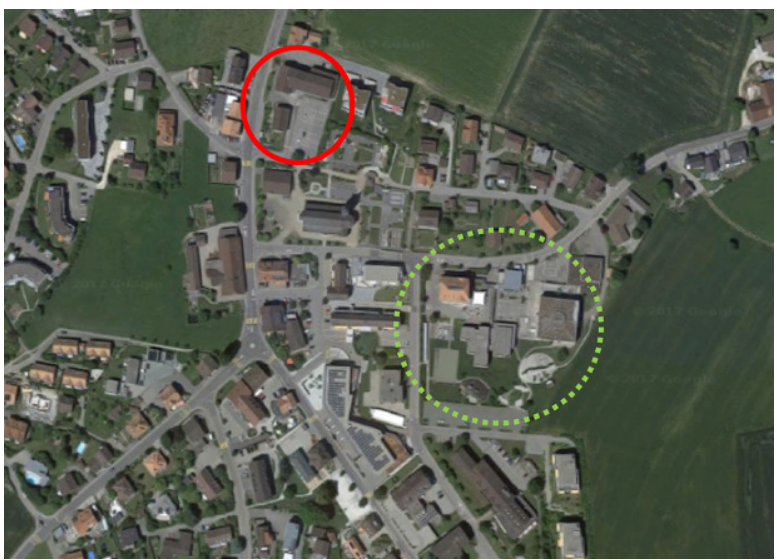


Dies steht vordergründig im Kontrast zur extravaganten Architektur des Hauses, das gesehen werden will. (Abb. 3).

Das Gebäude erhebt sich dreistöckig über einem quadratischen Grundriss. Charakteristisch ist, dass anstelle eines Treppenhauses der Kern des Hauses eine spiralförmige Rampe darstellt. Die Räume sind gleichförmig und werden als unregelmäBige Vierecke durch die Längs-, Breit- und Diagonal-Achsen gebildet. Sie befinden sich vertikal auf unterschiedlichen Ebenen. Die Fassade ist ein mit gelben Streifen bedruckter Glaskubus.

Das Gebäude ist sowohl in seiner postmodernen Formensprache als auch in seiner Materialität ein repräsentatives Gebäude. Als Fassadenhülle ist Glas anspruchsvoll und teuer. Es bringt jedoch eine Wahl zum Ausdruck, die ein hohes Level der Technikbeherrschung und eine vermögende Bauherrschaft sprichwörtlich spiegelt. Glas steht für den Nimbus von Innovation sowie Technik- und Naturbeherrschung. Als Glaskubus materialisiert das Haus Durchlässigkeit und Spiegelung: Es ist Selbstreferenz und Projektionsfläche der Bauherrschaft, die sich mit dem Gebäude ein Prestigeobjekt erstellte.

Das Schulhaus Gelb ist in Form und Gestalt nicht eindeutig als Schule erkennbar. Es könnte ebenso ein repräsentativer Museumsbau oder Sitz einer HightechFirma sein, die über Architektur eine werbewirksame Profilierung erreicht. Die aufsehenerregende Architektur ist damit nicht der Funktionalität der Schule geschuldet als vielmehr der Repräsentation der Bauherrschaft. Die Gemeinde affiziert mit dem Gebäude Urbanität, Innovation und Hightech und repräsentiert damit eine Transformation des dörflichen Charakters von Schmitten zu einer urban geprägten Agglomeration. Das Schulhaus wurde erbaut, damit es eine Wahrnehmung weit über den sichtbaren Perimeter hinaus erreicht. Der Repräsentationscharakter kann damit nicht „nur“ als identitätsstiftendes Merkmal einer vorhandenen Gemeinde gedeutet werden. Welches sind also die symbolischen Bedeutungs- und Sinnstrukturen, die sich die Gemeinde Schmitten mit dem Schulhaus Gelb errichtet?

Mit Bourdieu gedacht sind Schulbauten objektiviertes Kulturkapital. Zugleich sind sie symbolisches Kapital, die „Beherrschung von symbolischen Ressourcen, die auf Bekanntheit und Anerkennung beruhen" (Bourdieu 2011, S. 193). In dieser Lesart schreiben sich Schulhäuser als Statussymbole in einen sozialen Raum ein, in denen sich die Gemeinden distinktiv mittels ikonischer Architektur positionieren.

In der Schweiz ist dies besonders ausgeprägt, da es keine freie Schulwahl gibt. Schulen konkurrieren nicht um Schüler und Schülerinnen. Die Gemeinden in der Schweiz stehen jedoch in einem Standortwettbewerb um Steuersubstrate. Gerade weil es in der Schweiz keine Schulwahl gibt, ist der „Ruf“ einer Schule für diejenigen sozialen Schichten, die sich die „Wahl“ leisten können, relevant, da die „Schulwahl“ über die Wohnsitznahme erfolgt. Insbesondere Mittelschichteltern verfügen über ein feines Sensorium für die Einzugsgebiete der Schulkreise und -häuser. Daraus resultiert eine große soziale Segregation in den Schulen, die sich in den Raumbildern bzw. in einer sozio-ökonomischen räumlichen Segregation abbildet. Mit der Absenz eines Schulwahldiskurses haben die Schulen in der Schweiz, obwohl sie ,geleitete Schulen“ sind, wenig Autonomie und wenig Mittel für eine eigene Profilierung (siehe Hangartner und Svaton 2016). Dies gilt insbesondere in Bezug auf ihre Bausubstanzen, da die Bauaufgaben bei den Baudepartementen der 
Gemeinden angesiedelt sind, wobei keine oder nur wenig Mitwirkung der Schulen vorgesehen ist. In der Konsequenz davon wird der Schulhausbau von Architekten, Bauplanern und schulfernen Behörden dominiert. Als Resultat wird oft ,gute“ Architektur gleichsam als ,gute pädagogische“ Architektur betrachtet, ohne dass Letztere ausbuchstabiert werden müsste.

Für die Fragestellung von Interesse ist jedoch, dass der Schulhausbau für die Gemeinden die Möglichkeit darstellt, sich nach ihren Möglichkeiten selbst ein „Gesicht“" zu geben. Die Architektur des Schulhauses Gelb kann dahingehend interpretiert werden, dass die teure, exzentrische und ikonische Architektur eine dominante Repräsentation der Bauherrschaft darstellt, die eine Sendung über ihre Grenzen hinaus in Anspruch nimmt und sich damit in einem Wettbewerb positioniert. Denn wie Böhme und Herrmann (2011, S. 36) feststellen: „Die schulbauliche Beschaffenheit und Form stößt spezifische Milieus ab und zieht andere an, wie der Schulstandort oder die Schulregion auch.“ Dies wird in besonderem Maße für Grundschulen von Agglomerationen gelten, da sie bei der Wohnsitznahme von jungen Familien ein Entscheidungskriterium darstellen.

Festzuhalten ist an dieser Stelle, dass der Steuerwettbewerb und die Konkurrenz um vermögende Mittelschichtfamilien dem normativ-pädagogischen Diskurs entgegensteht: Die Homogenität des Sozialraums wird im schulpädagogischen Diskurs als unerwünscht bezeichnet (zur Diskussion siehe Krüger et al. 2020), und in der Schweiz wird gar diskutiert, eine Heterogenität der Schulen über neue „Grenzziehungen“ zu erreichen (siehe Dlabac und Amrhein 2019). Damit steht die Schulhausarchitektur in einem Spannungsverhältnis zwischen Repräsentation der Bauherrschaft und Funktionalität als Schule, wobei zumindest in Schmitten Erstere dominiert.

Verkürzt sei noch auf die implizit formativen Wirkungen des Schulhaus Gelb verwiesen. Glas als primärer Baustoff hat eine visuelle Transparenz zur Folge. Auch die klimatische Durchlässigkeit ist groß und macht eine vollautomatisierte, technische Klimaregulation und -steuerung notwendig. Ohne einen hohen Grad an Automation ist das Haus kaum nutzbar. Als sozialisatorischen Effekt impliziert das Schulhaus Gelb damit eine Normalität, in welcher die Verschränkung menschlicher Lebenspraxis mit einer durch Technik gesteuerten Umgebung den „Normalfall“ darstellt. Die sich selbst steuernde Umgebung ist dabei nicht nur technischer Natur, sondern setzt sich auch als sozial formativ wirkende Architektur fort. Die gläserne Transparenz impliziert ein Beobachtet-Sein, unabhängig davon, ob ein Beobachter faktisch anwesend ist oder nicht. Der Glaskubus perfektioniert damit die eines asymmetrischen Blick- und Kontrollregime eines Panoptikums. Bezeichnend dafür ist eine über Architektur erreichte (Selbst-)Kontrolle, indem ein potenzieller Beobachter antizipiert wird. Das Schulhaus entwickelt aus sich heraus eine disziplinierende Wirkung, die konformes Verhalten fördert und deviantes Verhalten tendenziell unterbindet.

Als sozialisatorische Implikation stellt das gläserne Haus eine Umgebung her, in der die Subjekte die Absenz von Privatsphäre als Normalität internalisieren. Das gläserne Haus versinnbildlicht damit den gläsernen Menschen, in welchem über vollständige Transparenz Disziplin, Selbst- und Affektkontrolle über Artefakte erzeugt oder vor allem auch aus sich heraus selbstgesteuert werden. 


\subsection{Schulraum als öffentlicher Raum für alle und die „Schulförmigkeit“ in Architekturen}

Außenräume sind typischerweise Pausenräume oder Räume, die sowohl als Orte formalisierten Unterrichts wie auch als Orte des Nicht-Unterrichts genutzt werden. Dabei sind Schulanlagen auch als außerschulische Sozialisationsräume von Belang. Schulaußenräume werden zunehmend geöffnet, da sie insbesondere in städtischen Siedlungen Residualräume darstellen, an denen sich Kinder und Jugendliche außerhalb der Schulzeit überhaupt noch treffen können. Im Schulbaudiskurs wird die Öffnung von Schulräumen überdies schon länger gefordert (z.B. Montag Stiftung 2011). Aus der Analyse des Schulareals Schmitten konnten drei dominante Strukturprinzipien rekonstruiert werden: Der Schulraum öffnet sich auch für Nicht-Schüler und -Schülerinnen; der öffentliche Raum ist nach dem Vorbild urbaner Räume gestaltet; alle Nutzer und Nutzerinnen werden durch die im Raum platzierten Artefakte wie Schüler und Schülerinnen in einer analogen „schulförmigen“ Weise adressiert. Dies soll im Folgenden ausformuliert werden.

Der Außenraum (vgl. Abb. 5) besteht aus zwei leeren, monotonen Grundflächen (Rasen und Verbundsteine). Auf diesen sind die Gebäude und unterschiedliche Funktionsfelder angeordnet. Letztere stehen jeweils für sich und sind über einen Rundweg miteinander verbunden. Die räumlich getrennten Funktionsflächen richten sich an spezifische Akteursgruppen und sind mit entsprechenden architektonischen Artefakten möbliert: Sport- und Skateboardinganlage, Spielplätze, Grillstellen, ein Jugendtreff u.a.m. Es kann festgehalten werden, dass sich der Schulraum mit dieser Gestaltung öffnet und im Prinzip ein Angebot für alle Lebenslagen zur Verfügung stellt, die mit Kindern und Jugendlichen im Zusammenhang stehen. Zugleich wird eine Separierung analog der Alterskohorten in der Schule in räumlich getrennte Bereiche konstruiert.

Unter ästhetischen und funktionalen Gesichtspunkten stellt die Außenanlage der Schule Schmitten ein Abbild eines urbanen Stadtparks und damit eines urbanen Lebensstils dar. Als Bedeutungsstruktur reproduziert sich, dass der Außenraum nicht nur als Freizeit- und Sozialisationsraum für Kinder gedacht ist, sondern ausnahmslos als artifiziell geschaffener Raum konzipiert wird. Unstrukturierte oder wenig strukturierte Flächen gibt es lediglich in Form der „leeren“ Rasenflächen. „Natur“ ist ausschließlich in kultivierter domestizierter Form vorzufinden: als wohlgeordnete und beschnittene Bepflanzungen, als Spielgeräte in einer „naturnahen“ $̈$ Ästhetik, die ausgewaschenen Baumstämmen in einem Kiesbett gleichen u.a.m. Ansonsten prägen Gebilde von Lifestylesportarten und Orte vorstrukturierter Handlungen das Bild. Dies hat durchaus auch sozialisatorische Wirkungen. Wie beim Schulhaus Gelb ist eine menschlich und durch Technik gesteuerte (Um-)Welt die Normalform. Auch das Picknick oder der Grillausflug ins „Grüne“ wird durch architektonische Artefakte vorstrukturiert und dem Raum eingraviert.

Die Bedeutung des Schulraums als Sozialisationsraum zeigt sich darin, dass ein artifizieller Raum geschaffen wird, der mit konstruierten Gebilden möbliert ist. Damit sind es Dinge, die explizit von Erwachsenen für Kinder und für den schulischen Gebrauch hergestellt wurden und denen ,erzieherische Dingfunktionen“(Nohl 2011, S. 125 ff.) innewohnen. Erzieherische Funktionen sind an Dinge delegiert, insofern 
Kinder sich durch Übung den ,richtigen“ Gebrauch der Dinge bzw. die Handlungsprogramme von Dingen aneignen. Gemeinsam ist den „Möblierungen“, dass es Dinge sind, die in ihrem Aufforderungsgehalt eine spezifische, nämlich „schulförmige“ Struktur haben (ausführlich X, S. $290 \mathrm{ff}$.). Darunter wird verstanden, dass durch Artefakte und Architekturen monofunktionale und spezifisch gerichtete Handlungsaufforderungen erzeugt werden: z. B. Balancieren, Schaukeln u.a.m. Zudem sind die Artefakte so konstruiert, dass jeweils nur die intendierte Handlung ausgeführt werden kann - z. B. das Balancieren auf einem Baumstamm - und alle anderen Aneignungen unterbunden werden.

Dinge haben ,deontische Macht“, d.h. sie verkörpern Regeln, die in ihnen institutionalisiert sind (vgl. Searl 2012). Die Übungen des korrekten Gebrauchs und das Erlernen der Bedeutung von Dingen z. B. als Geldschein ist Teil des Bildungsprozesses. Bemerkenswert erscheint mir die Form. Denn als sozialisatorischer Effekt von schulförmig strukturierten Objekten ist anzunehmen, dass implizit eine „Gefolgschaft“ gegenüber den „Handlungsprogrammen“ der Dingen einsozialisiert wird. Hier reproduziert sich die Sinnstruktur der Handlungsaufforderungen bzw. der formativen Wirkungen von Schulhausarchitekturen: Der Schulraum stellt sich als ein arrangierter Raum dar, der spezifische, in der Regel monofunktionale und eindeutige Praktiken aus sich heraus erzeugt und steuert. Die sozialisatorischen Wirkungen sind dabei auch in einer Erziehung über Dinge zu sehen: Das Arrangement des schulischen Außenraums als Spielraum sieht vor, dass die Subjekte den institutionalisierten Regeln der Artefakte folgen und nicht, dass sie sich die Welt selbstständig kreativ anzueignen und sich aktiv in Zusammenhänge einbringen.

\section{Schlussbetrachtung}

Schulräume sind zentrale Sozialisationsräume, wenn es um die Inkorporation sozialer und die Herausbildung mentaler Strukturen geht. Als äußere soziale Realitäten stellen sie den Aneignungsraum dar, dessen Strukturen von Kindern internalisiert und inkorporiert werden. Die Analysen zweier sehr unterschiedlicher Gebäude zeigt, dass Schulen in ihren Raumordnungen und Architekturen eine in die Zukunft projizierte Normalität vorwegnehmen: Schulhäuser sind formative Architekturen. Damit sind sie tatsächlich Bildungsbauten bzw. gebaute Bildung nicht nur als Hüllen von staatlich organisierten Lernorten und von Bildungsprozessen, sondern auch als Sozialisationsinstanz und ,dritter Erzieher“ (Brüschweiler und Reutlinger 2014).

Auch als Symbolträger sind Schulhäuser im Siedlungsbild herausragende Bauten mit formativem Charakter für die umgebende Siedlung und für die Subjekte, die sich darin sozialisieren. Wie Bourdieu ausführt, ist „,der Soziale Raum (...) zugleich in die Objektivität der räumlichen Strukturen eingeschrieben und in die subjektiven Strukturen, die zum Teil aus der Inkorporation dieser objektivierten Strukturen hervorgehen“ (Bourdieu 1991, S. 28). Schulhäuser als symbolische Repräsentationsbauten von Staatlichkeit nehmen nicht nur physisch verortet eine besondere Stellung im Siedlungsgefüge ein. Sie gravieren sich in den sozialen Raum ein und sind zugleich Werkzeug, diesen zu beschreiben. 
In der historischen Entwicklung hat sich der Schulraum sukzessive ausgedehnt. Dies ist räumlich-material der Fall, indem sich die Schulanlage auch den NichtSchülern und Nicht-Schülerinnen öffnet. Dabei ist auch eine Entgrenzung zu konstatieren: Die Schule entwickelt sich von einem Ort der sekundären Sozialisation und einer spezifischen pädagogischen Praxis zu einem Sozialisationsort auch in Zeiten des Nicht-Unterrichts. Zudem findet eine Entgrenzung auch dadurch statt, dass Nicht-Schüler adressiert werden. Sowohl in der Architektursprache als auch in den funktionalen „Angeboten“ der Schulanlage als öffentlicher Stadtpark wird eine spezifische soziale Gruppe angesprochen. Es ist nicht primär die autochthone Bevölkerung, sondern es sind potenzielle Neuzuzüger, die in der Stadt keinen bezahlbaren Wohnraum finden. In erster Linie werden Familien einer urban orientierten Mittelschicht mit Kindern im Grundschulalter angesprochen.

In den Analysen architektonischer Artefakte und Schulgebäuden realisiert sich die schulische Sozialisation in einem formativen „schulförmigen“ Strukturprinzip. Die untersuchten Primarschulen stellen in ihren Architekturen Räume her, die auf die Internalisierung von monofunktionalen Routinen gerichtet sind, da sie eindeutige Lernprozesse emergieren und gleichzeitig Aneignung und offene Bildungsprozesse unterbinden. Dies steht dem pädagogischen Diskurs entgegen, der Lern- und Bildungsräume fordert, die vielseitige Anregungen bieten. Es steht aber auch der naturwüchsigen Sozialisation entgegen, in denen Kinder die Welt häufig gerade invers zur intendierten Aneignung nutzen (Muchow 2012).

Als Prämisse eines architektursoziologischen Vorgehens wurde ausgeführt, dass Architekturen als unbewusstes, dauerhaft stabiles „Gedächtnis“ fungieren und handlungsleitend wirken. Das Prinzip der Schulförmigkeit ist, so die aus der architektursoziologischen Analyse bestimmte These, dass sich die „Schulförmigkeit“ als Grundmotiv in den Lehrmitteln, Arbeitsblättern und schulischen Artefakten wiederfindet. So wie sich die Formen, z. B. die Rechteckigkeit der Klassenzimmer und Schulräume, in der Rechteckigkeit fast sämtlicher Schulmaterialien wiederfindet, findet sich auch der latent wirkende Grundstein der „Schulförmigkeit“" als prägende Vorstellung eindeutiger, gerichteter Lernprozesse in den pädagogisch-didaktischen Schulmaterialien wieder. Empirisch ist dies systematisch zu überprüfen. Auch gilt dies nicht ausschließlich für die Grundschule. Die „Schulförmigkeit“" scheint sowohl im „Raumbild“ als auch im ,Traumbild der Gesellschaft“ als latent wirkendes Strukturprinzip festgeschrieben. In der Grundschulpädagogik erhält sie aber eine besondere Bedeutung, da unter sozialisatorischen Gesichtspunkten die Grundschulpädagogik für die Internalisierung gesellschaftlicher Prinzipien grundlegend ist. Die Frage ist, ob damit Subjekte sozialisiert werden, die die Problemlösekapazitäten der Gesellschaft erhöhen, die sich selbstständig, selbsttätig und kreativ in Handlungs- und Interaktionskontexte einbringen und ihr Autonomiepotenzial einlösen. Oder wird damit viel eher eine Gefolgschaft gegenüber Dingen habitualisiert, indem deren Handlungsaufforderungen zu ,selbst gewählten“ Handlungseinschränkungen gedeutet werden und die schlussendlich vor allem konformes Verhalten erzeugt.

Funding Open access funding provided by FHNW University of Applied Sciences and Arts Northwestern Switzerland 
Open Access Dieser Artikel wird unter der Creative Commons Namensnennung 4.0 International Lizenz veröffentlicht, welche die Nutzung, Vervielfältigung, Bearbeitung, Verbreitung und Wiedergabe in jeglichem Medium und Format erlaubt, sofern Sie den/die ursprünglichen Autor(en) und die Quelle ordnungsgemäß nennen, einen Link zur Creative Commons Lizenz beifügen und angeben, ob Änderungen vorgenommen wurden.

Die in diesem Artikel enthaltenen Bilder und sonstiges Drittmaterial unterliegen ebenfalls der genannten Creative Commons Lizenz, sofern sich aus der Abbildungslegende nichts anderes ergibt. Sofern das betreffende Material nicht unter der genannten Creative Commons Lizenz steht und die betreffende Handlung nicht nach gesetzlichen Vorschriften erlaubt ist, ist für die oben aufgeführten Weiterverwendungen des Materials die Einwilligung des jeweiligen Rechteinhabers einzuholen.

Weitere Details zur Lizenz entnehmen Sie bitte der Lizenzinformation auf http://creativecommons.org/ licenses/by/4.0/deed.de.

\section{Literatur}

Alkemeyer, T. (2011). Die Körperlichkeit des Lernens, der Bildung und der Subjektivierung. In I. Erler, et al. (Hrsg.), Wie Bourdieu in die Schule kommt (S. 55-68). Innsbruck, Wien, Bozen: Studien Verlag.

Barth, H. (2004). Im Schatten der Moderne. Gustav Lüdecke 1890-1976. Rekonstruktion eines Architektenwerkes. Dortmund: Dortmunder Vertrieb für Bau- und Planungsliteratur.

Becker-Lenz, R., Franzmann, A., Jansen, A., \& Jung, M. (Hrsg.). (2016). Die Methodenschule der Objektiven Hermeneutik: Eine Bestandsaufnahme. Wiesbaden: Springer VS.

Böhme, J., \& Herrmann, I. (2011). Schule als pädagogischer Machtraum. Typologie schulischer Raumentwürfe. Wiesbaden: VS.

Bourdieu, P. (1991). Physischer, sozialer und angeeigneter physischer Raum. In M. Wentz (Hrsg.), Stadt-Räume (S. 25-34). Frankfurt a. M.: Campus.

Bourdieu, P. (2011). Das ökonomische Feld. In Der Einzige und sein Eigenheim (S. 185-222). Hamburg: VSA.

Brüschweiler, B., \& Reutlinger, C. (2014). Raum als dritter Erzieher. In U. Deinet \& C. Reutlinger (Hrsg.), Tätigkeit-Aneignung-Bildung (S. 175-188). Wiesbaden: Springer.

Caruso, M. (2003). Biopolitik im Klassenzimmer: Zur Ordnung der Führungspraktiken in den Bayerischen Volksschulen (1869-1918). Weinheim: Beltz.

Dlabac, O., \& Amrhein, A. (2019). Durchmischung in städtischen Schulen - eine politische Aufgabe? https://www.zdaarau.ch/dokumente/SB-17-Durchmischung-Schulen-ZDA.pdf. Zugegriffen: 30. März 2020.

Durkheim, É. (2003). Erziehung, Moral und Gesellschaft: Vorlesung an der Sorbonne 1902/1903. Frankfurt a. M.: Suhrkamp.

Egger, J. (2019). Häuser machen Schule. Eine architektursoziologische Analyse gebauter Bildung. Wiesbaden: Springer VS.

Epstein, J. L., \& Salinas, K. C. (2004). Partnering with families and communities. Educational Leadership, $61(8), 12-18$.

Fischer, J. (2017). Gebaute Welt als schweres Kommunikationsmedium der Gesellschaft. Architektur und Religion aus architektursoziologischer Perspektive. In U. Karstein \& T. Schmidt-Lux (Hrsg.), Architekturen und Artefakte (S. 49-69). Wiesbaden: Springer VS.

Foucault, M. (2012). Die Mittel der guten Abrichtung. In U. Bauer, U.H. Bittlingmayer \& A. Scherr (Hrsg.), Handbuch Bildungs- und Erziehungssoziologie (S. 199-212). Wiesbaden: Springer VS.

Halbwachs, M. (1997). La mémoire collective. Paris: Albin Michel.

Hangartner, J., \& Svaton, C. J. (2016). „Geleitete Schule“ zwischen Profilierung und Vereinheitlichung. In J. Hangartner \& M. Heinzer (Hrsg.), Gemeinden in der Schul-Governance der Schweiz (S. 199-220). Wiesbaden: Springer VS.

Helfenberger, M. (2016). Schulhausbau in Zürich von 1860 bis 1920 - zwischen Expertenherrschaft und öffentlicher Kontrolle. In J. Hangartner \& M. Heinzer (Hrsg.), Gemeinden in der Schul-Governance der Schweiz (S. 221-247). Wiesbaden: Springer VS.

Kogler, R. (2015). Zonen, Inseln, Lebenswelten, Sozialräume. Konzepte zur Raumaneignung im Alltag von Kindern. In J. Scheiner \& C. Holz-Rau (Hrsg.), Räumliche Mobilität und Lebenslauf (S. 43-56). Wiesbaden: Springer VS. 
Kracauer, S. (1930). Über Arbeitsnachweise. http://raumgegenzement.blogsport.de/2009/10/23/siegfriedkracauer-ueber-arbeitsnachweise-1930/. Zugegriffen: 25. Okt. 2019.

Krüger, J. O., Roch, A., \& Breidenstein, G. (2020). Schulwahl als Elitebildung? Zur Bearbeitung des Segregationsverdachts im elterlichen Diskurs. In J. O. Krüger, A. Roch \& G. Breidenstein (Hrsg.), Szenarien der Grundschulwahl (Bd. 70, S. 41-56). Wiesbaden: Springer VS.

Montag Stiftung Jugend und Gesellschaft Bonn (2011). Schulen planen und bauen. Grundlagen und Prozesse. Berlin:: Jovis.

Muchow, M. (2012). Der Lebensraum des Großstadtkindes. Weinheim, Basel: Beltz Juventa.

Nohl, A.-M. (2011). Pädagogik der Dinge. Bad Heilbrunn: Julius Klinkhardt.

Perlick, P. (1969). Architektur im Dienste der Pädagogik. Ein Beitrag zur Planung von Grund- und Hauptschulen sowie verwandten Systemen. Wuppertal: Aloys Henn.

Pestalozzi, J.H. (2010). An die Unschuld, den Ernst und den Edelmuth meines Zeitalters und meines Vaterlandes: Ein Wort der Zeit. http://www.e-helvetica.nb.admin.ch/directAccess?callnumber=nbdig33998. Zugegriffen: 14. Apr. 2016.

Piaget, J. (2017). Das Weltbild des Kindes. Stuttgart: Klett-Cotta.

Schäfers, B. (2010). Architektursoziologie. Grundlagen-Ansätze-Belege. In P. Trebsche, et al. (Hrsg.), Der gebaute Raum. Bausteine einer Architektursoziologie vormoderner Gesellschaften (S. 29-40). Münster: Waxmann.

Schmidtke, O. (2006). Architektur als professionalisierte Praxis. Soziologische Fallrekonstruktionen zur Professionalisierungsbedürftigkeit der Architektur. Frankfurt a. M.: Humanities online.

Schmidtke, O. (2008). Soziologische Architekturinterpretation mit Hilfe der Objektiven Hermeneutik. Zum Interpretieren von Architektur. Wolkenkuckucksheim, 12(2).

Schneeberger, E. (2005). Schulhäuser für Stadt und Land. Der Volksschulhausbau im Kanton Bern am Ende des 19. Jahrhunderts. Bern: Historischer Verein des Kantons Bern.

Searl, J. R. (2012). Wie wir die soziale Welt machen. Berlin: Suhrkamp.

Simmel, G. (1908). Der Raum und die räumlichen Ordnungen der Gesellschaft. In G. Simmel (Hrsg.), Soziologie. Untersuchungen über die Formen der Vergesellschaftung (S. 460-526). Frankfurt am Main: Duncker \& Humblot.

Steets, S. (2015). Der sinnhafte Aufbau der gebauten Welt. Eine Architektursoziologie. Berlin: Suhrkamp. Zinnecker, J. (Hrsg.). (1975). Der heimliche Lehrplan. Untersuchungen zum Schulunterricht. Weinheim, Basel: Beltz. 